\title{
Search for new light vector boson using $J / \Psi$ at BESIII and Belle II
}

\author{
Kayoung Ban, Yongsoo Jho, Youngjoon Kwon, Seong Chan Park, Seokhee Park \\ and Po-Yan Tseng \\ Department of Physics and IPAP, Yonsei University, \\ Seoul 03722, Republic of Korea \\ E-mail: ban94gy@yonsei.ac.kr, jys34@yonsei.ac.kr, \\ yjkwon63@yonsei.ac.kr, sc.park@yonsei.ac.kr, \\ seokhee.park@yonsei.ac.kr, tpoyan1209@gmail.com
}

ABSTRACT: We investigate various search strategies for light vector boson $X$ in $\mathcal{O}(10) \mathrm{MeV}$ mass range using $J / \Psi$ associated channels at BESIII and Belle II: (i) $J / \Psi \rightarrow \eta_{c} X$ with $10^{10} \mathrm{~J} / \Psi$ s at BESIII, (ii) $J / \Psi\left(\eta_{c}+X\right)+\ell \bar{\ell}$ production at Belle II, and (iii) $J / \Psi+X$ with the displaced vertex in $X \rightarrow e^{+} e^{-}$decay are analyzed and the future sensitivities at Belle II with $50 \mathrm{ab}^{-1}$ luminosity are comprehensively studied. By requiring the displaced vertex to be within the beam pipe, the third method results in nearly background-free analysis, and the vector boson-electron coupling and the vector boson mass can be probed in the unprecedented range, $10^{-4} \leq\left|\varepsilon_{e}\right| \leq 10^{-3}$ and $9 \mathrm{MeV} \leq m_{X} \leq 100 \mathrm{MeV}$ with $50 \mathrm{ab}^{-1}$ at Belle II. This covers the favored signal region of ${ }^{8} \mathrm{Be}^{*}$ anomaly recently reported by Atomki experiment with $m_{X} \simeq 17 \mathrm{MeV}$.

Keywords: Beyond Standard Model, Gauge Symmetry

ARXiv EPrint: 2012.04190 


\section{Contents}

1 Introduction 1

2 Effective Lagrangian $\quad 2$

3 Signal and background from $J / \Psi \rightarrow \eta_{c} e^{+} e^{-} \quad 3$

4 The $e^{+} e^{-} \rightarrow \ell^{+} \ell^{-}+J / \Psi \rightarrow \ell^{+} \ell^{-} e^{+} e^{-} \eta_{c}$ at Belle II $\quad 7$

5 The $e^{+} e^{-} \rightarrow X+J / \Psi \rightarrow e^{+} e^{-}+J / \Psi$ displaced vertex at Belle II 9

6 Summary and conclusion $\quad 12$

\section{Introduction}

The Standard Model (SM) is a successful theory describing physics at least up to the electroweak scale, having survived more than 40 years of various experimental tests. However, there still remain a handful number of experimental and observational claims that indicate discrepancies from the SM predictions and consequently request extension of the SM: nonzero mass of neutrinos [1], anomalous magnetic moment of muon, $(g-2)_{\mu}[2,3]$, existence of dark matter (DM) [4], ${ }^{1}$ and baryon vs. antibaryon asymmetry of the universe $[6,7]$. There have been discussions of extending the SM by gauging the lepton number, e.g. $L_{\mu}-L_{\tau}$ or $L_{e}-L_{\tau}$ [8], intending to explain DM $[9,10]$, the muon anomalous magnetic moment $(g-2)_{\mu}[11-14]$, and more recently EDGES $21 \mathrm{~cm}$ anomaly [15]. The extension gives rise to a leptophilic light vector boson, dubbed as $X$ in this paper. We note that the $X$ boson may couple to the quarks via interactions with unknown heavy fermions that mix with SM quarks [16]. It may then be responsible for the recent anomaly from the KOTO experiment in $K_{L} \rightarrow \pi^{0} \nu \bar{\nu}$ [17-20] and also the anomaly from the Atomki experiment in both ${ }^{8} \mathrm{Be}^{*}$ and ${ }^{4} \mathrm{He}^{*}$. The preferred mass of $X$ for these cases is in sub-GeV range; in particular $m_{X} \simeq 17 \mathrm{MeV}$ for Atomki [21, 22].

High luminosity lepton colliders provide ideal environments to test for such light $X$ boson. Thanks to less severe QCD backgrounds, the lepton colliders have definite advantages over hadron colliders even when the $X$ boson has feeble couplings with the SM particles. In this paper, we take the lepton colliders, BESIII and Belle II, and study the search strategies of $X$. In particular, we focus on the channels in association with a $J / \Psi$ meson, which will be enormously produced at BESIII and also at Belle II, thereby leaving the signals of $X$ in various channels:

- At BESIII, up to now, $10^{10} \mathrm{~J} / \Psi$ events are collected, thus provide an excellent probe to study the $J / \Psi$ rare decays to the $X$ boson.

\footnotetext{
${ }^{1}$ Primordial black holes may explain the whole DM. See, e.g., [5].
} 
- At Belle II, even though less number of $J / \Psi$ are expected, we use the process $e^{+} e^{-} \rightarrow$ $\ell^{+} \ell^{-} J / \Psi \rightarrow \ell^{+} \ell^{-} \eta_{c} X \rightarrow \ell^{+} \ell^{-} \eta_{c} e^{+} e^{-}(\ell=e$ or $\mu)$, in which $J / \Psi$ and $\eta_{c}$ are inferred by the recoil masses of $\ell^{+} \ell^{-}$and $\ell^{+} \ell^{-} e^{+} e^{-}$, respectively.

- At Belle II, we also use the channel $e^{+} e^{-} \rightarrow X+J / \Psi$ where the $X$ bosons will leave signals with displaced vertices. Due to higher center-of-mass (CM) energy at Belle II, the $X$ boson will be boosted and travel several millimeters before it decays into $e^{+} e^{-}$.

The rest of this paper is dedicated to studying the sensitivity reach of finding $X$ boson taking realistic experimental situations into account under the effective field theory framework.

This paper is organized as follows: we first set up our theoretical framework and introduce the effective interactions in section 2. The analysis for BESIII is carried out in section 3. In section 4 and $5, e^{+} e^{-} \rightarrow \ell^{+} \ell^{-} J / \Psi$ and $e^{+} e^{-} \rightarrow X+J / \Psi$ with displaced vertex signal are analyzed, respectively for Belle II. Finally, our results are summarized in section 6 .

\section{Effective Lagrangian}

The vectorlike interactions of the $X$ boson with the SM fermions, $f$, are introduced by the effective Lagrangian:

$$
\mathcal{L} \supset-e X_{\mu} \sum_{f} \varepsilon_{f} \bar{f} \gamma^{\mu} f
$$

where we regard the couplings $\varepsilon_{f}$ as free parameters without knowing the origin. In particular, we will assume four universal couplings, $\varepsilon_{u}, \varepsilon_{d}, \varepsilon_{e}$, and $\varepsilon_{\nu}$, for up-type quarks, down-type quarks, charged leptons, and neutrinos, respectively, in our analysis below. We also note that the new interactions do not induce any axial anomaly by construction.

If the new boson $X$ is responsible for the recent Atomki anomaly [22] via the process ${ }^{8} \mathrm{Be}+X \rightarrow{ }^{8} \mathrm{Be}+e^{+} e^{-}$, its mass should be $m_{X} \simeq 17 \mathrm{MeV}$ and the couplings with the first generation quarks should be in a particular window [23-25]:

$$
\left|\varepsilon_{u}+\varepsilon_{d}\right| \simeq 3.7 \times 10^{-3} .
$$

From the NA48/2 experiment for $\pi^{0} \rightarrow X \gamma$, we require a protophobic condition [26]:

$$
\left|2 \varepsilon_{u}+\varepsilon_{d}\right|<8 \times 10^{-4} \text {. }
$$

Taking both relations in eq. 2.2 and eq. 2.3 into account, we finally get the preferred value for up-type and down-type quark couplings:

$$
\varepsilon_{u} \simeq \pm 3.7 \times 10^{-3}, \varepsilon_{d} \simeq \mp 7.4 \times 10^{-3},
$$

which we will rely on below.

The coupling to the leptons, especially to electron and electron-neutrino, are stringently constrained by the beam dump experiment SLAC E141 [27], the anomalous magnetic moment of the electron $g-2$ [28], and neutrino-electron scattering experiment [29]:

$$
\begin{aligned}
4.2 \times 10^{-4} \lesssim\left|\varepsilon_{e}\right| & \lesssim 1.4 \times 10^{-3}, \\
\sqrt{\varepsilon_{e} \varepsilon_{\nu}} & \lesssim 7 \times 10^{-5} .
\end{aligned}
$$


When a small coupling for neutrino $\varepsilon_{\nu} \ll 10^{-6}$ is assumed, we do not worry about constraints from neutrinos.

\section{Signal and background from $J / \Psi \rightarrow \eta_{c} e^{+} e^{-}$}

In the SM, the decay $J / \Psi \rightarrow \eta_{c} \gamma^{*} \rightarrow \eta_{c} e^{+} e^{-}$is radiatively allowed [30]. Its partial width is expressed with the form factor $f_{\mathrm{VP}}(0)$ for on-shell photon at the vanishing momentum transfer limit $q^{2}=0[31]$

$$
\Gamma\left(J / \Psi \rightarrow \eta_{c} \gamma\right)=\frac{1}{3} \frac{\alpha_{\mathrm{EM}}\left(m_{J / \Psi}^{2}-m_{\eta_{c}}^{2}\right)^{3}}{8 m_{J / \Psi}^{3}}\left|f_{\mathrm{VP}}(0)\right|^{2},
$$

from which the form factor $\left|f_{\mathrm{VP}}(0)\right|=0.68949 \mathrm{GeV}^{-1}$ is determined with the fine structure constant $\alpha_{\mathrm{EW}} \simeq 1 / 137$, the masses of $J / \Psi$ and $\eta_{c}, m_{J / \Psi}=3.0969 \mathrm{GeV}$, and $m_{\eta_{c}}=$ $2.9839 \mathrm{GeV}$, respectively, and the measured width $\Gamma\left(J / \Psi \rightarrow \eta_{c} \gamma\right)=1.5793 \mathrm{keV}[7,32-34]$. The form factor $f_{\mathrm{VP}}\left(q^{2}\right)$ for general $q^{2} \neq 0$ is obtained by $F_{\mathrm{VP}}\left(q^{2}\right) \equiv f_{\mathrm{VP}}\left(q^{2}\right) / f_{\mathrm{VP}}(0)=$ $1 /\left(1-\frac{q^{2}}{\Lambda^{2}}\right)$ from the pole approximation with pole mass $\Lambda=m_{\psi^{\prime}}=3.686097 \mathrm{GeV}$ for $J / \Psi$.

The normalized differential widths for partial decay widths of $J / \Psi \rightarrow \eta_{c} \gamma^{*} \rightarrow \eta_{c} e^{+} e^{-}$ and $J / \Psi \rightarrow \eta_{c} X^{*} \rightarrow \eta_{c} e^{+} e^{-}$, respectively for off-shell photon and $X$ boson are obtained using a common factor $F_{\mathrm{VP}}\left(q^{2}\right)[30]$ :

$$
\begin{aligned}
& \frac{d \Gamma_{\eta_{c} \gamma^{*}}}{d q^{2} \Gamma_{J / \Psi \rightarrow \eta_{c} \gamma}}=\left|F_{\mathrm{VP}}\left(q^{2}\right)\right|^{2} \times F_{\mathrm{QED}}\left(q^{2}\right) \\
& \frac{d \Gamma_{\eta_{c} X^{*}}}{d q^{2} \Gamma_{J / \Psi \rightarrow \eta_{c} \gamma}}=\left|F_{\mathrm{VP}}\left(q^{2}\right)\right|^{2} \times F_{X}\left(q^{2}\right),
\end{aligned}
$$

where the kinematic window is given as $\left(2 m_{e}\right)^{2} \leq q^{2}=m_{e^{+} e^{-}}^{2} \leq\left(m_{J / \Psi}-m_{\eta_{c}}\right)^{2}$. Since the mass difference between $J / \Psi$ and $\eta_{c}$ is only $113 \mathrm{MeV}$, it is hard to significantly boost $X$ boson in this channel and produce displaced-vertex signal in a collider detector.

The precise expression for the factor $F_{\mathrm{QED}}$ is shown in ref. [30] where the factor is found to include the amplitude square and phase space factor for off-shell photon. Analogous expression for $F_{X}$ is obtained:

$$
\begin{aligned}
F_{X}\left(q^{2}\right)= & \frac{\alpha_{\mathrm{EM}}\left(\varepsilon_{c} \cdot \varepsilon_{e}\right)^{2}}{3 \pi}\left(\frac{q^{2}}{\left[\left(q^{2}-m_{X}^{2}\right)^{2}+m_{X}^{2} \Gamma_{X}^{2}\right]}\right) \\
& \times\left(1-\frac{4 m_{e}^{2}}{q^{2}}\right)^{1 / 2}\left(1+\frac{2 m_{e}^{2}}{q^{2}}\right)\left[\left(1+\frac{q^{2}}{m_{J / \Psi}^{2}-m_{\eta_{c}}^{2}}\right)^{2}-\frac{4 m_{J / \Psi}^{2} q^{2}}{\left(m_{J / \Psi}^{2}-m_{\eta_{c}}^{2}\right)^{2}}\right]^{3 / 2}
\end{aligned}
$$

by replacing the couplings and propagator from $F_{\mathrm{QED}}$.

Assuming that $\varepsilon_{\nu} \ll 10^{-6}$ and $\varepsilon_{e} \simeq 10^{-3}$, and the quark channels are kinematically forbidden with $m_{X} \leq 2 m_{\pi}$, the $X$ boson dominantly decay to electrons with the width

$$
\Gamma_{X \rightarrow e^{+} e^{-}}=\frac{\varepsilon_{e}^{2} \alpha_{\mathrm{EM}} m_{X}}{3}\left(1+\frac{2 m_{e}^{2}}{m_{X}^{2}}\right) \sqrt{1-\frac{4 m_{e}^{2}}{m_{X}^{2}}},
$$

which is narrow $\Gamma_{X} \ll m_{X}$. 


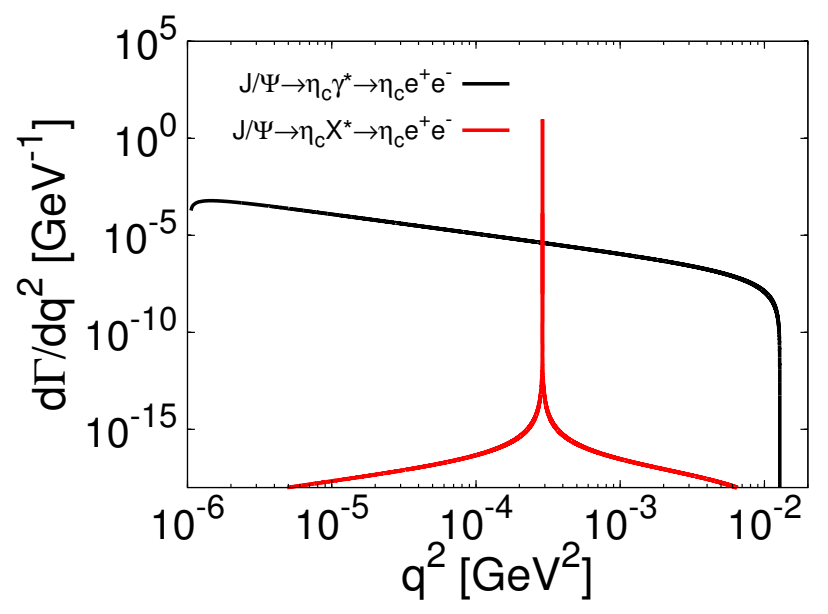

Figure 1. The $e^{+} e^{-}$invariant mass distributions of signal $J / \Psi \rightarrow \eta_{c} X^{*} \rightarrow \eta_{c} e^{+} e^{-}$(red) and background $J / \Psi \rightarrow \eta_{c} \gamma^{*} \rightarrow \eta_{c} e^{+} e^{-}$(black), where $q^{2} \equiv m_{e^{+} e^{-}}^{2}$. Input parameters are $m_{X}=$ $17 \mathrm{MeV}, \varepsilon_{c}=3.7 \times 10^{-3}$, and $\varepsilon_{e}=10^{-3}$.

After performing the integration of $q^{2}$, we can obtain the partial decay width $\Gamma_{\eta_{c} X^{*}}$. By inserting favoured coupling values $\varepsilon_{c}=\varepsilon_{u}=3.7 \times 10^{-3}, \varepsilon_{e}=10^{-3}$ and fixing $m_{X}=17 \mathrm{MeV}$ for ${ }^{8} \mathrm{Be}^{*}$ anomaly, it gives $\Gamma_{\eta_{c} \gamma^{*}}=2.09 \times 10^{-5} \mathrm{keV}$ and implies

$$
\operatorname{Br}\left(J / \Psi \rightarrow \eta_{c} X^{*} \rightarrow \eta_{c} e^{+} e^{-}\right)=1.64 \times 10^{-6}\left(\frac{\varepsilon_{c}}{10^{-2}}\right)^{2},
$$

which is about three orders of magnitude smaller than that of the $\eta_{c} \gamma^{*}$ background, $\operatorname{Br}\left(J / \Psi \rightarrow \eta_{c} \gamma^{*} \rightarrow \eta_{c} e^{+} e^{-}\right)=1.03 \times 10^{-4}{ }^{2}$

The $e^{+} e^{-}$invariant-mass-squared distributions for signal $\left(\eta_{c} X^{*}\right)$ and background $\left(\eta_{c} \gamma^{*}\right)$ are compared in figure 1 . The different features are clearly displayed: the signal has a peak at $q^{2}=m_{X}^{2}$ and the background is broadly distributed. Therefore our task now is to efficiently extract the signal near the peak and suppress the background.

We first impose a kinematic condition for signal: $M_{e^{+} e^{-}} \subset\left[\left(m_{X}-\sigma_{m}\right),\left(m_{X}+\sigma_{m}\right)\right]$, where $\sigma_{m}$ is the $e^{+} e^{-}$mass resolution which is roughly of the same order of magnitude as the energy resolution $\sigma_{E}$. The signal yield $S$ and background yield $B$ are now obtained as

$$
\begin{aligned}
& S=N_{J / \Psi} \times \frac{\int_{\left(m_{X}-\sigma_{m}\right)^{2}}^{\left(m_{X}\right.} d q^{2} \frac{d \Gamma_{\eta_{c} X^{*}}}{d q^{2}}}{\Gamma_{J / \Psi}}, \\
& B=N_{J / \Psi} \times \frac{\int_{\left(m_{X}-\sigma_{m}\right)^{2}}^{\left(m_{X}+\sigma_{m}\right.} \frac{d \Gamma_{\eta_{c} \gamma^{*}}}{d q^{2}}}{\Gamma_{J / \Psi}},
\end{aligned}
$$

where $N_{J / \Psi}$ is the total number of $J / \Psi$ produced in the collision, and $\Gamma_{J / \Psi}=92.9 \mathrm{keV}$ is the total decay width of $J / \Psi[7]$.

\footnotetext{
${ }^{2}$ In the background estimation, the other background sources are not considered. For instance, $\gamma \rightarrow e^{+} e^{-}$ conversion near the detector materials is contributed with $J / \Psi \rightarrow \eta_{c} \gamma$ decay as a background source. The process is suppressed by examining the vertex position of the resulting $e^{+} e^{-}$. For the vertexing performance of Belle II, the reader is directed to ref. [35].
} 


\begin{tabular}{|cc|}
\hline Branching Ratio & Detection efficiency \\
\hline $\operatorname{Br}\left(\eta_{c} \rightarrow K^{+} K^{-} \pi^{0}\right)=(1.15 \pm 0.12) \%$ & $18.82 \%$ \\
$\operatorname{Br}\left(\eta_{c} \rightarrow K_{S}^{0} K^{ \pm} \pi^{\mp}\right)=(2.60 \pm 0.21) \%$ & $21.22 \%$ \\
$\operatorname{Br}\left(\eta_{c} \rightarrow 2\left(\pi^{+} \pi^{-} \pi^{0}\right)\right)=(15.2 \pm 1.8) \%$ & $3.07 \%$ \\
\hline
\end{tabular}

Table 1. The branching fractions of $\eta_{c}$ decay modes with corresponding efficiencies.

\begin{tabular}{|c|ccccc|}
\hline & $\sigma_{m}=1 \mathrm{MeV}$ & $\sigma_{m}=2 \mathrm{MeV}$ & $\sigma_{m}=5 \mathrm{MeV}$ & $\sigma_{m}=10 \mathrm{MeV}$ & $\sigma_{m}=15 \mathrm{MeV}$ \\
\hline$S$ & 188 & 263 & 277 & 277 & 277 \\
$B$ & 3686 & 7399 & 18989 & 42436 & 87640 \\
\hline$S / \sqrt{B}$ & 3.10 & 3.06 & 2.01 & 1.34 & 0.94 \\
\hline
\end{tabular}

Table 2. For $N_{J / \Psi}=10^{11}$ and favoured parameters $\varepsilon_{c}=\varepsilon_{u}=3.7 \times 10^{-3}, \varepsilon_{e}=10^{-3}, m_{X}=17 \mathrm{MeV}$ for ${ }^{8} \mathrm{Be}^{*}$ anomaly, the significances of signal to background from $J / \Psi \rightarrow \eta_{c} e^{+} e^{-}$with various energy resolutions of detector and $1.23 \% \eta_{c}$ reconstruction efficiency.

The BESIII experiment, which has collected $10^{10} \mathrm{~J} / \Psi$ events in the resonance process $e^{+} e^{-} \rightarrow J / \Psi$ [30], plans to increase the size of $J / \Psi$ sample to $10^{11}$ events in the near future. The energy resolution of BESIII is $\sigma_{E} / E \simeq 0.005$ for the final-state electron, which smears the invariant mass distribution of $e^{+} e^{-}$by $\sigma_{m} \simeq 1 \mathrm{MeV}$.

In order to exclusively reconstruct the $J / \Psi \rightarrow \eta_{c} e^{+} e^{-}$decays, we have to consider $\eta_{c}$ decay modes that can be fully reconstructed with reasonable background contamination. Table 1 lists the branching fractions of a few such $\eta_{c}$ decay modes along with the corresponding efficiencies [36].

The overall efficiency $\epsilon$ of the above three $\eta_{c}$ modes is obtained by adding the individual efficiencies weighted by their corresponding branching fractions: $\epsilon=1.23 \%$. Given these, and taking $17 \mathrm{MeV} X$ boson for ${ }^{8} \mathrm{Be}^{*}$ anomaly to be real, we list, in table 2, the expected significances of $J / \Psi \rightarrow \eta_{c} X \rightarrow \eta_{c} e^{+} e^{-}$with $N_{J / \Psi}=10^{11}$ at BESIII under the assumption of $\varepsilon_{c}=\varepsilon_{u}$, for various $\sigma_{m}$ values.

For general light vector boson searches through $J / \Psi \rightarrow \eta_{c} e^{+} e^{-}$, the variation of the expected significance over $\left(m_{X}, \varepsilon_{c}, \varepsilon_{e}\right)$ are shown in figure 2 . With the present value of $N_{J / \Psi}=10^{10}$ at BESIII, the region of sensitivity is $\left|\varepsilon_{c}\right| \gtrsim 5 \times 10^{-3}$ at $m_{X} \simeq 17 \mathrm{MeV}$ as shown in the upper left panel of figure 2 and left panel of figure 3. The sensitivity slightly improves as $m_{X}$ increases, because of the reduction of background (see figure 1), and reaches the maximal sensitivity $\left|\varepsilon_{c}\right| \gtrsim 3 \times 10^{-3}$ at $m_{X} \simeq 60 \mathrm{MeV}$. But as $m_{X}$ approaches $m_{J / \Psi}-m_{\eta_{c}}$, the sensitivity becomes weaker due to the phase space suppression. The two right panels of figure 2 show that the significance is independent of the $\varepsilon_{e}$ as we expect from the narrow width approximation. For $N_{J / \Psi}=10^{11}$ which is expected in the near future, the projected sensitivity becomes $\left|\varepsilon_{c}\right| \gtrsim 3 \times 10^{-3}$ at $m_{X} \simeq 17 \mathrm{MeV}$ as shown in the bottom-left panel of figure 2 and the right panel of figure 3 , whereby the entire favored region of ${ }^{8} \mathrm{Be}^{*}$ anomaly can be probed.

An alternative way to explicitly reconstructing $\eta_{c}$ in $J / \Psi \rightarrow \eta_{c} e^{+} e^{-}$at BESIII is to use the recoil of $e^{+} e^{-}$. As the $e^{ \pm}$carries low energy around $50 \mathrm{MeV}$, it gets difficult 

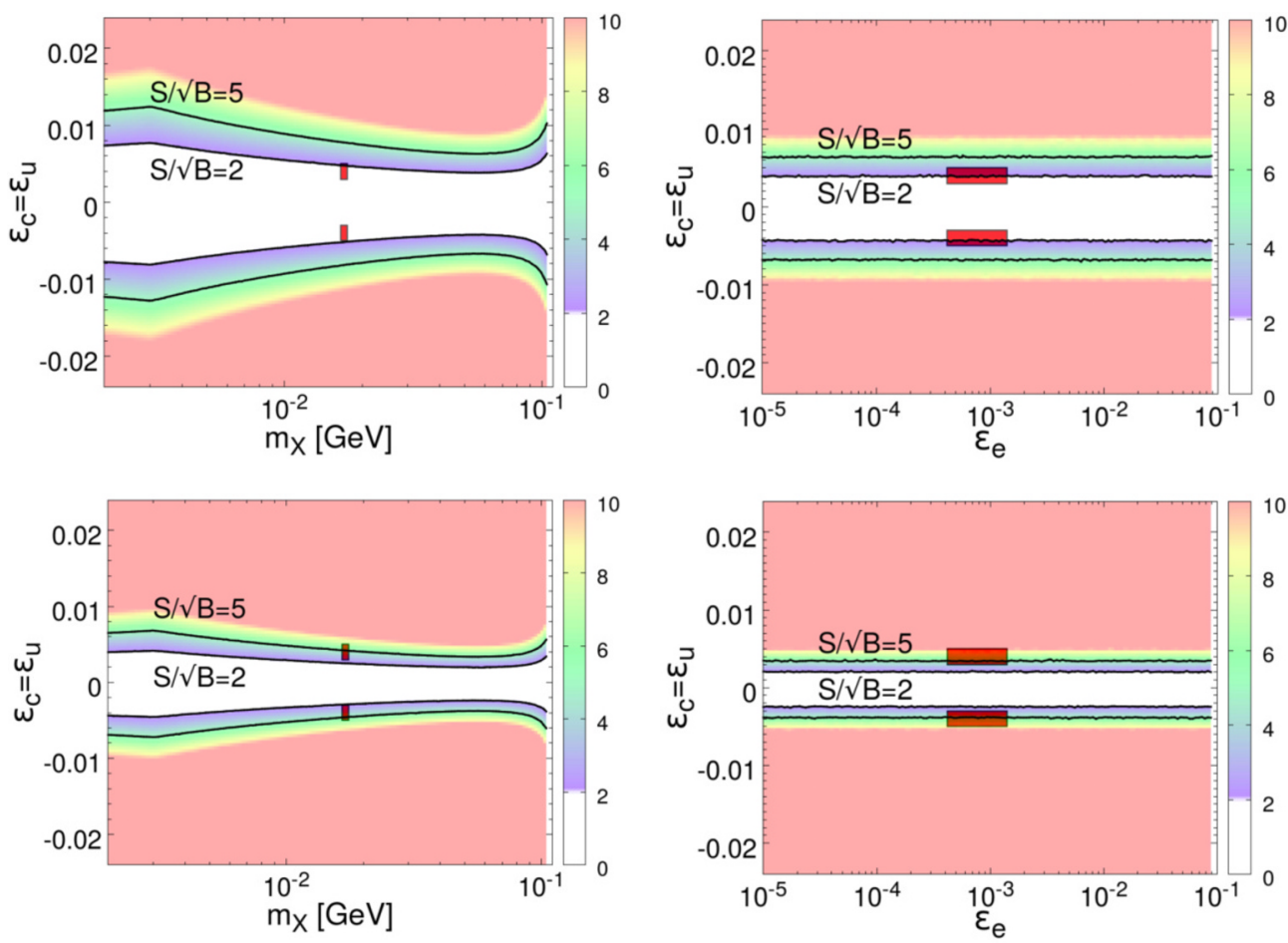

Figure 2. The significance $S / \sqrt{B}$ on $\left(m_{X}, \varepsilon_{c}\right)$ and $\left(\varepsilon_{c}, \varepsilon_{e}\right)$ planes from $J / \Psi \rightarrow \eta_{c} e^{+} e^{-}$light $X$ vector boson searches at BESIII. Adopt $N_{J / \Psi}=10^{10}$ (upper panels) and $N_{J / \Psi}=10^{11}$ (bottom panels), reconstructed efficiency of $\eta_{c}$ from table 1 , and the invariant mass cut $\left|M_{e e}-m_{X}\right| \leq \sigma_{m}=$ $2 \mathrm{MeV}$. The red boxes indicate the preferred regions for ${ }^{8} \mathrm{Be}^{*}$ anomaly.
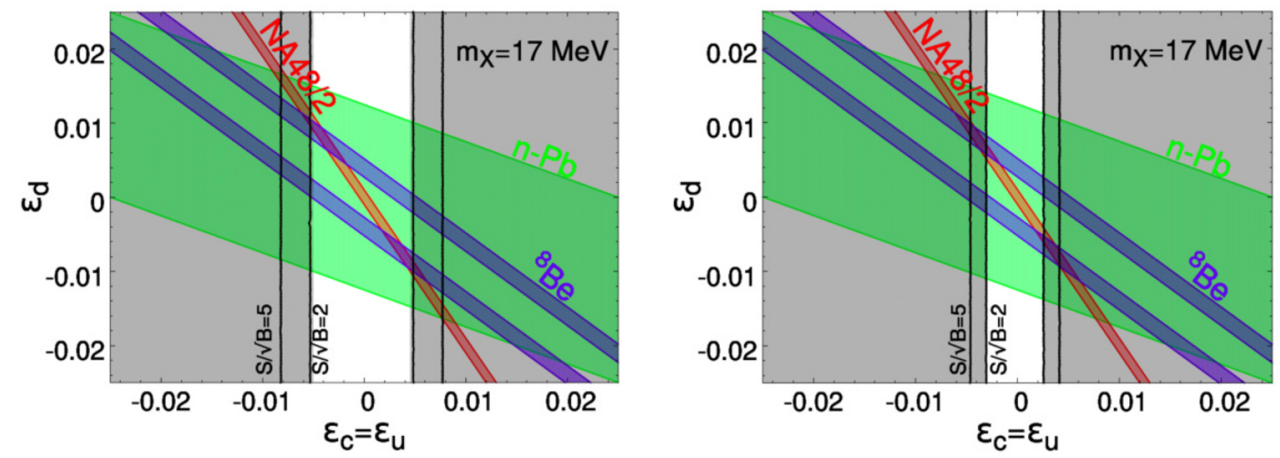

Figure 3. The current (left-panel: $N_{J / \Psi}=10^{10}$ ) and future (right-panel: $N_{J / \Psi}=10^{11}$ ) BESIII sensitivities by assuming the reconstruction efficiencies from table 1 and taking the invariant mass cut $\left|M_{e e}-m_{X}\right| \leq \sigma_{m}=2 \mathrm{MeV}$. Compare with the allowed regions for ${ }^{8} \mathrm{Be}^{*}$ anomaly, NA48/2 for $\pi^{0}$ decay [26] and neutron-nucleus scattering [37]. 


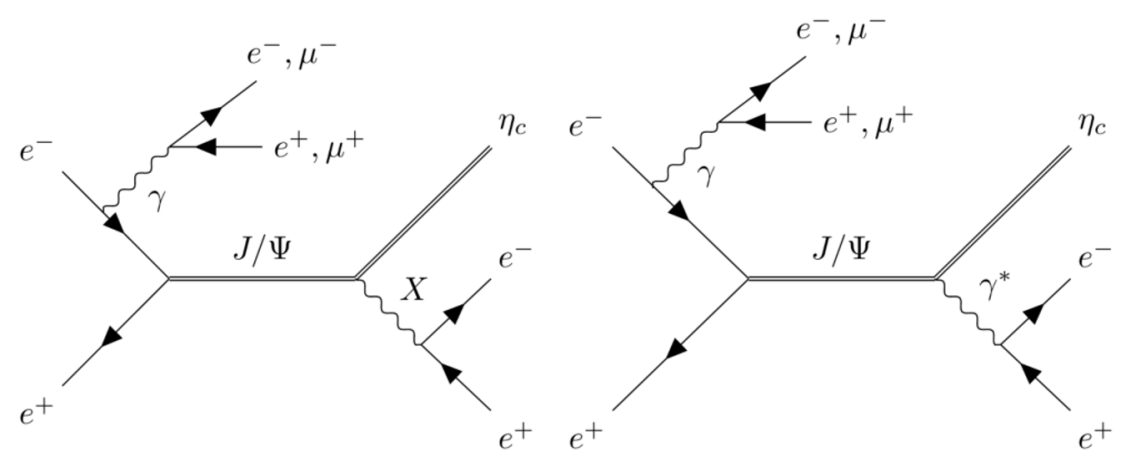

Figure 4. The Feynman diagrams of the signal (left) and background (right).

to distinguish $e^{ \pm}$tracks from $\pi^{ \pm}$background. With an improvement of low-energy electron identification in the future, the BESIII with $N_{J / \Psi}=10^{11}$ can reach the sensitivity of $\left|\varepsilon_{c}\right| \simeq 10^{-3}$.

\section{The $e^{+} e^{-} \rightarrow \ell^{+} \ell^{-}+J / \Psi \rightarrow \ell^{+} \ell^{-} e^{+} e^{-} \eta_{c}$ at Belle II}

For vector meson $J / \Psi$, the partial width to $e^{+} e^{-}$is given by

$$
\Gamma_{J / \Psi \rightarrow e^{+} e^{-}}=\frac{g_{J / \Psi e e}^{2}}{12 \pi} m_{J / \Psi}\left(1+\frac{2 m_{e}^{2}}{m_{J / \Psi}^{2}}\right) \sqrt{1-\frac{4 m_{e}^{2}}{m_{J / \Psi}^{2}}},
$$

where $g_{J / \Psi e e}=8.2048 \times 10^{-3}[7]$ is the coupling strength in the effective interaction $g_{J / \Psi e e}\left[\bar{e} \gamma^{\mu} e\right](J / \Psi)_{\mu}$ that matches the measured value $\Gamma_{J / \Psi \rightarrow e^{+} e^{-}}=5.53 \mathrm{keV}$ [7].

Then the cross sections to $\ell^{+} \ell^{-} J / \Psi$ where $\ell=e$, or $\mu$ at Belle II are obtained via $e^{+} e^{-} \rightarrow \gamma^{*} J / \Psi$ :

$$
\begin{gathered}
\sigma\left(e^{+} e^{-} \rightarrow \gamma^{*}+J / \Psi \rightarrow e^{+} e^{-} J / \Psi\right)=286 \mathrm{fb}, \\
\sigma\left(e^{+} e^{-} \rightarrow \gamma^{*}+J / \Psi \rightarrow \mu^{+} \mu^{-} J / \Psi\right)=58.4 \mathrm{fb} .
\end{gathered}
$$

With the design integrated luminosity $\mathcal{L}=50 \mathrm{ab}^{-1}$, we estimate $N_{J / \Psi}=1.75 \times 10^{7}$ events for $e^{+} e^{-} \rightarrow \gamma^{*}+J / \Psi \rightarrow \ell^{+} \ell^{-} J / \Psi$ at Belle II. This $N_{J / \Psi}$ is applied to eq. 3.5, along with eq. 4.1, to give estimates of $S$ and $B$ :

$$
\begin{aligned}
& S=\mathcal{L} \times \sigma\left(e^{+} e^{-} \rightarrow \ell^{+} \ell^{-} J / \Psi\right) \times \operatorname{Br}\left(J / \Psi \rightarrow \eta_{c} X^{*} \rightarrow \eta_{c} e^{+} e^{-}\right) \simeq 28.2\left(\frac{\varepsilon_{c}}{10^{-2}}\right)^{2}, \\
& B=\mathcal{L} \times \sigma\left(e^{+} e^{-} \rightarrow \ell^{+} \ell^{-} J / \Psi\right) \times \operatorname{Br}\left(J / \Psi \rightarrow \eta_{c} \gamma^{*} \rightarrow \eta_{c} e^{+} e^{-}\right) \simeq 1772 .
\end{aligned}
$$

Therefore, the estimated $S / \sqrt{B}$ is too small at this level so that we will improve the analysis by a more realistic MC study below.

For event generation, we use MG5_AMC@NLO [38] for both signal and background with FeynRules v2.0 [39] model for $J / \Psi, \eta_{c}$ mesons and $X$ boson couplings and $X$ couples to the leptons. We generate with the $E_{\mathrm{beam} 1,2}=5.2941 \mathrm{GeV}$ in the CM frame, which is 

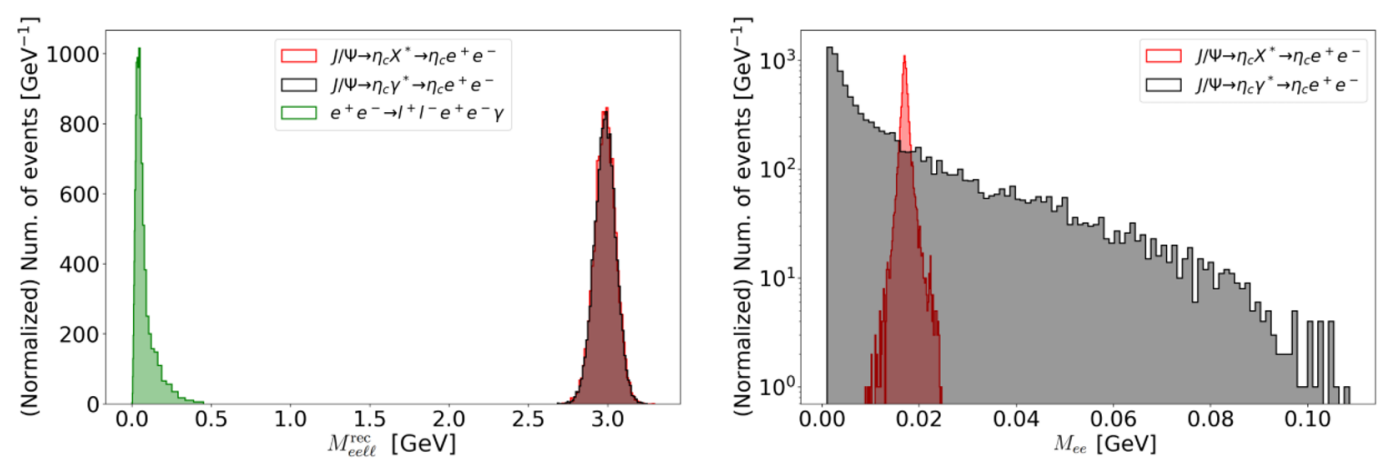

Figure 5. The $e^{+} e^{-} \ell^{+} \ell^{-}$recoil mass (left) and $e^{+} e^{-}$invariant mass (right) distributions for the parton level Monte-Carlo simulation data with the smearing effect. Input parameter is $m_{X}=$ $17 \mathrm{MeV}$. Here, we normalized to $10^{5}$ events for each channel.

boosted by $\beta=0.2732$ with respect to the lab frame. The amplitude of the electromagnetic Dalitz decay, $V \rightarrow P e^{+} e^{-}$can be written in a Lorentz-invariant form [30],

$$
T\left(V \rightarrow P e^{+} e^{-}\right)=4 \pi \alpha_{\mathrm{EM}} f_{\mathrm{VP}} \epsilon^{\mu \nu \rho \sigma} p_{\mu} q_{\nu} \epsilon_{\rho} \frac{1}{q^{2}} \bar{u}_{1} \gamma_{\sigma} \nu_{2}
$$

and we can obtain the interaction Lagrangian as,

$$
\begin{aligned}
\mathcal{L} \supset & f_{\mathrm{VP}}\left(-2 \sqrt{\pi \alpha_{\mathrm{EM}}} \partial_{\mu} P \partial_{\nu} V_{\rho} \epsilon^{\mu \nu \rho \sigma} A_{\sigma}-g_{X c} \partial_{\mu} P \partial_{\nu} V_{\rho} \epsilon^{\mu \nu \rho \sigma} X_{\sigma}\right) \\
& -g_{e V} \bar{e} \gamma^{\mu} e V_{\mu}-g_{X e} \bar{e} \gamma^{\mu} e X_{\mu},
\end{aligned}
$$

where $g_{X c}, g_{X e}$, and $g_{e V}$ are the effective coupling constants, whose numerical values are to be obtained by experiments.

Similar to the BESIII, photon conversion process where the photon from $J / \Psi \rightarrow \eta_{c} \gamma$ hits the beam pipe or vertex detector layers and converts to $e^{+} e^{-}$can be a background source, but they are controlled by examining the $e^{+} e^{-}$vertex position. Another possible background is $e^{+} e^{-} \rightarrow \ell^{+} \ell^{-}+$(anything) where (anything) may contain a number of lowmomentum charged particles that are misidentified as electrons. However, this process does not show peak in the $M_{\ell^{+} \ell^{-}}^{\text {recil }} \simeq M_{J / \Psi}$ variable, and can be suppressed by inspecting the $M_{\ell^{+} \ell^{-}}^{\text {recoil }}$ distribution. In the figure 5, the recoil mass of $e^{+} e^{-} \ell^{+} \ell^{-}$and invariant mass distributions of $e^{+} e^{-}$are plotted for the signal (shown in red), and the $\ell^{+} \ell^{-} e^{+} e^{-}(\gamma)$ (green) and $J / \Psi \rightarrow \eta_{c} \gamma^{*}$ (gray) backgrounds, where we produce equal number of events $\left(=10^{5}\right)$ for each sample at the parton level before applying any selection cuts. We give a Gaussian smearing effect with the momentum resolution $\sigma_{p_{\ell^{ \pm}}} / p_{\ell^{ \pm}}=0.005$ on the parton level data for our analysis. To simulate the effects of the Belle II detector, we apply the following baseline cuts: $\left|\eta_{\ell^{ \pm}}^{*}\right| \leq 1.60$ in the CM frame $[14,40],\left|E_{\mu^{ \pm}}\right| \geq 0.6 \mathrm{GeV}$, and $\left|E_{e^{ \pm}}\right| \geq 0.06 \mathrm{GeV}$ in the lab frame [35]. Note that we require very low energy threshold for electrons so as to keep most of the signals, because the $e^{+} e^{-}$from $J / \Psi \rightarrow \eta_{c} e^{+} e^{-}$are very soft. This inevitably would cause large background from pion tracks being misidentified as electrons. ${ }^{3}$

\footnotetext{
${ }^{3}$ Currently, the most powerful observable for electron identification (e-ID) at Belle II is the $E / p$ where
} 


\begin{tabular}{|c|c|c|}
\hline Cuts & $\mathrm{B}$ & $\mathrm{S}$ \\
\hline Processes & $\eta_{c} \gamma^{*} \rightarrow \eta_{c} e e$ & $\eta_{c} X \rightarrow \eta_{c} e e$ \\
\hline & 100000 & 100000 \\
Baseline Cuts & 7170 & 6290 \\
$\left|M_{e e \ell \ell}^{\mathrm{rec}}-m_{\eta_{c}}\right| \leq 200 \mathrm{MeV}$ & 7071 & 6219 \\
$\left|M_{e e}-m_{X}\right| \leq 2 \mathrm{MeV}$ & $\mathbf{3 7 7}$ & $\mathbf{5 8 8 0}$ \\
\hline
\end{tabular}

Table 3. Signal and background events of $e^{+} e^{-} \rightarrow \ell^{+} \ell^{-} e^{+} e^{-} \eta_{c}$ after cuts at Belle II.

\begin{tabular}{|c|ccc|}
\hline Luminosity & $50 \mathrm{ab}^{-1}$ & $100 \mathrm{ab}^{-1}$ & $200 \mathrm{ab}^{-1}$ \\
\hline$\left|\varepsilon_{c}\right|$ & $\gtrsim 1.76 \times 10^{-2}$ & $\gtrsim 1.48 \times 10^{-2}$ & $\gtrsim 1.24 \times 10^{-2}$ \\
\hline
\end{tabular}

Table 4. Sensitivities on $\varepsilon_{c}$ of $17 \mathrm{MeV} X$ boson from $\ell^{+} \ell^{-} J / \Psi \rightarrow \ell^{+} \ell^{-} e^{+} e^{-} \eta_{c}$ search at Belle II with luminosities 50,100 , and $200 \mathrm{ab}^{-1}$. Here we require $S / \sqrt{B}=2$.

And we give two kinematic requirements: for the energy-momentum 4-vector of $\eta_{c}$, we use the energy and momentum recoiling against $e^{+} e^{-} \ell^{+} \ell^{-}$. The signal and background distributions of the recoil mass $M_{e e \ell}^{\text {rec }}$, smeared by the charged-track momentum resolution, are displayed in the left panel of figure 5. The $J / \Psi \rightarrow \eta_{c} e^{+} e^{-}$events clearly show a peak

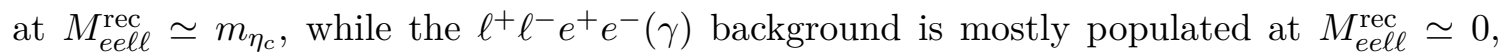
therefore we require $\left|M_{\text {eell }}^{\text {rec }}-m_{\eta_{c}}\right| \leq 200 \mathrm{MeV}$ to eliminate the $\ell^{+} \ell^{-} e^{+} e^{-}(\gamma)$ background. In addition, we apply $\left|M_{e e}-m_{X}\right| \leq 2 \mathrm{MeV}$, whereby the $\eta_{c} \gamma^{*}$ background from the process shown in the right panel of figure 4 are suppressed. In table 3 , we summarize the cumulative effects of the baseline cuts and the kinematic requirements.

The sensitivities of $\ell^{+} \ell^{-} e^{+} e^{-}$search at Belle II are derived from the requirement of $S / \sqrt{B}=2$. Combining eq. (4.3) and table 3 , we obtain the corresponding values of $\varepsilon_{c}$ with respect to luminosities of 50,100 , and $200 \mathrm{ab}^{-1}$ in table 4 . They are about 5 times larger than the estimates from current BESIII sensitivity $\left|\varepsilon_{c}\right| \gtrsim 5 \times 10^{-3}$ in section 3 .

\section{The $e^{+} e^{-} \rightarrow X+J / \Psi \rightarrow e^{+} e^{-}+J / \Psi$ displaced vertex at Belle II}

The $X$ boson produced in the process $e^{+} e^{-} \rightarrow X+J / \Psi$ travels several millimeters before decaying into $e^{+} e^{-}$in the Belle II detector and leaves displaced vertex. In particular, when the distance of the flight is between $2 \mathrm{~mm}$ and $8 \mathrm{~mm}$, which is inside the beam pipe, and outside the interaction region, Belle II has excellent power to reconstruct the displaced vertex and makes the signal almost free from the SM background [43]. Therefore, we propose to use the clean displaced $e^{+} e^{-}$vertex from the $X$ boson decay (along with prompt $\ell^{+} \ell^{-}$from $\left.J / \Psi\right)$.

The leading-order Feynman diagram of the relevant process is shown in the left panel of figure 6. A typical event with displaced vertex at Belle II detector with the $\ell^{+} \ell^{-}$from $J / \Psi$ decay is schematically shown in the right panel of figure 6 .

$E$ is the energy measured in the electromagnetic calorimeter and $p$ is the magnitude of 3-momentum measured in the drift chamber. [41] With low-momentum tracks that cannot reach the calorimeter [42], the performance of $e$-ID will thus degrade. For the actual data analysis, we encourage the Belle II collaboration to improve $e$-ID for low-momentum tracks along with systematic uncertainties. 

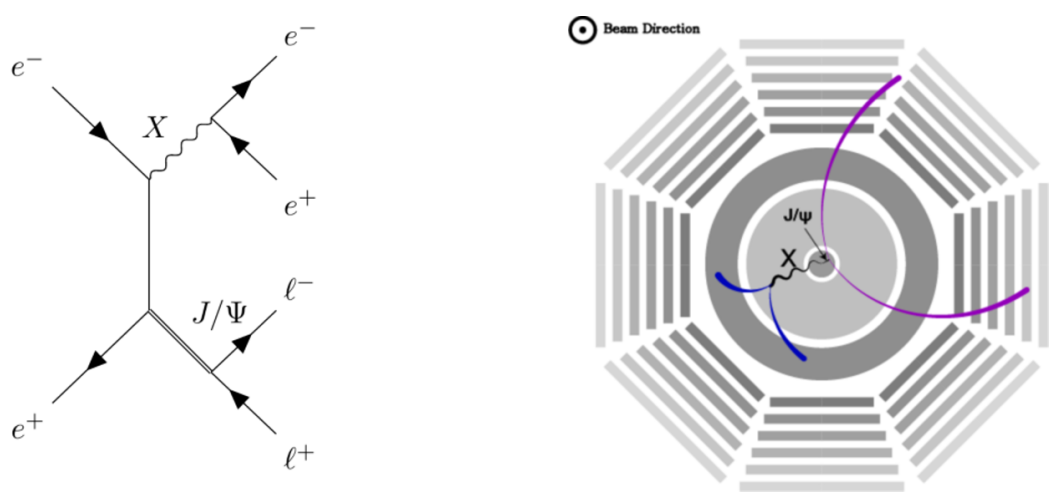

Figure 6. (Left panel) The Feynman diagram of the $e^{+} e^{-} \rightarrow X+J / \Psi \rightarrow e^{+} e^{-} \ell^{+} \ell^{-}$signal. (Right panel) The schematic picture of the decay process of the long-lived $X$ boson in the Belle II detector.
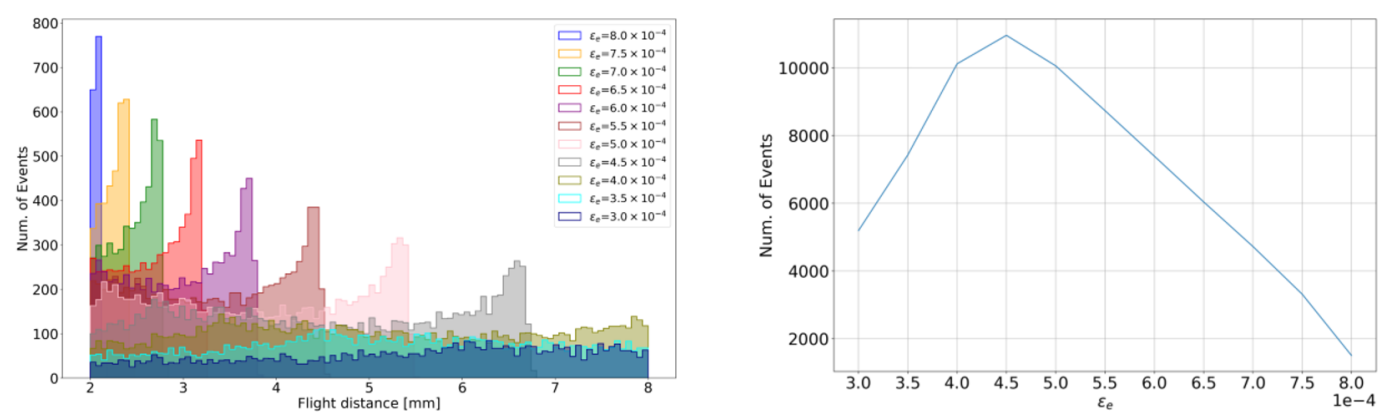

Figure 7. (Left panel) The transverse flight distance $d_{x y}$ of $17 \mathrm{MeV} X$ boson for $\varepsilon_{e}$ in $0.5 \times 10^{-4}$ steps from $3 \times 10^{-4}$ to $8 \times 10^{-4}$; (Right panel) The number of events that pass the baseline cuts and satisfy $2 \mathrm{~mm} \leq d_{x y} \leq 8 \mathrm{~mm}$ from the sample of $10^{5}$ generated signal events.

We note that compared with other lighter vector mesons, the heavier mass of $J / \Psi$ helps to induce larger scattering angle in such a way that more events from $X \rightarrow e^{+} e^{-}$ will satisfy the cut $\left|\eta_{\ell^{ \pm}}^{*}\right| \leq 1.60$. Furthermore, the electron and positron from $X \rightarrow e^{+} e^{-}$ carry energy above $\mathrm{GeV}$, which make them easier to be distinguished from charged-pion backgrounds.

The other advantage of this channel is that the signal strength only depends on the $\varepsilon_{e}$ coupling since only $X-e^{+}-e^{-}$vertices are involved at tree level. For $0.3 \times 10^{-3} \leq$ $\varepsilon_{e} \leq 0.8 \times 10^{-3}$, it yields a few $\mathrm{mm}$ transverse flight distance $d_{x y}$ which is defined as the distance between the beam axis and the $X$ decay vertex. The left-panel of figure 7 shows the distribution of $d_{x y}$ corresponding to several values of $\varepsilon_{e}$.

With the baseline cuts and $2 \mathrm{~mm} \leq d_{x y} \leq 8 \mathrm{~mm}$, we estimate the signal sensitivity by considering two cases: (i) explicitly reconstructing $J / \Psi \rightarrow \ell^{+} \ell^{-}$(' $e^{+} e^{-} \ell^{+} \ell^{-}$channel'), and (ii) using the recoil mass of $X \rightarrow e^{+} e^{-}$to infer $J / \Psi$ (' $e^{+} e^{-}$channel'). Tables 5 and 6 show, respectively for the $e^{+} e^{-} \ell^{+} \ell^{-}$and $e^{+} e^{-}$channels, the signal efficiencies and expected significances for various assumed values of $\varepsilon_{e}$, according to the $50 \mathrm{ab}^{-1}$ luminosity 


\begin{tabular}{|c|c|c|c|c|c|c|c|}
\hline$\varepsilon_{e} / 10^{-4}$ & 8.0 & 7.0 & 6.0 & 5.0 & 4.5 & 4.0 & 3.0 \\
\hline Baseline Cuts $(\%)$ & 13.8 & 13.8 & 13.8 & 13.8 & 13.8 & 13.8 & 13.8 \\
\hline $2 \mathrm{~mm}<d_{x y}<8 \mathrm{~mm}(\%)$ & 1.5 & 4.7 & 7.4 & 10.1 & 11.0 & 10.1 & 5.2 \\
\hline$N_{S}$ & 1.60 & 3.85 & 4.42 & 4.18 & 3.69 & 2.69 & 0.78 \\
\hline Significance $(B=0.1)$ & $2.4 \sigma$ & $4.6 \sigma$ & $5.0 \sigma$ & $4.8 \sigma$ & $4.5 \sigma$ & $3.6 \sigma$ & $1.5 \sigma$ \\
\hline Significance $(B=1)$ & $1.6 \sigma$ & $2.9 \sigma$ & $3.2 \sigma$ & $3.1 \sigma$ & $2.8 \sigma$ & $2.3 \sigma$ & $1.2 \sigma$ \\
\hline
\end{tabular}

Table 5. (From top rows to bottom) The fraction of events (in \%) that survive the baseline cuts mentioned in section 4 , and the flight distance $2 \mathrm{~mm} \leq d_{x y} \leq 8 \mathrm{~mm}$ cuts, for several values of $\varepsilon_{e}$ of $17 \mathrm{MeV} X$ boson; the number of signal events $N_{S}$ in the $e^{+} e^{-} \ell^{+} \ell^{-}$channel at Belle II with $50 \mathrm{ab}^{-1}$ luminosity; and the expected significances assuming 1 event, and 0.1 event of the SM background in the analysis channel after all cuts.

\begin{tabular}{|c|c|c|c|c|c|c|c|}
\hline$\varepsilon_{e} / 10^{-4}$ & 8.0 & 7.0 & 5.0 & 4.0 & 3.0 & 2.0 & 1.0 \\
\hline Baseline Cuts(\%) & 17.6 & 17.6 & 17.6 & 17.6 & 17.6 & 17.6 & 17.6 \\
\hline $2 \mathrm{~mm}<d_{x y}<8 \mathrm{~mm}(\%)$ & 1.6 & 5.3 & 12.3 & 12.9 & 7.4 & 2.3 & 0.5 \\
\hline$N_{S}$ & 14.6 & 35.7 & 42.7 & 28.7 & 9.23 & 1.28 & 0.07 \\
\hline Significance $(B=0.1)$ & \multicolumn{5}{|c|}{$>5 \sigma$} & $2.2 \sigma$ & $0.4 \sigma$ \\
\hline Significance $(B=1)$ & \multicolumn{5}{|c|}{$>5 \sigma$} & $1.6 \sigma$ & $0.9 \sigma$ \\
\hline
\end{tabular}

Table 6. The same as table 5, but using the $e^{+} e^{-}$channel.

at Belle II and the $e^{+} e^{-} \rightarrow X+J / \Psi$ cross section

$$
\sigma\left(e^{+} e^{-} \rightarrow X+J / \Psi\right)=2.77 \times 10^{-2} \times\left(\frac{\varepsilon_{e}}{10^{-3}}\right)^{2} \mathrm{fb} .
$$

The significances are calculated from the expected $p$-value of background-only hypothesis for each case.

The final result of expected sensitivity with Belle II at $50 \mathrm{ab}^{-1}$ luminosity is shown in figure 8. Also displayed in figure 8 are the expected results with the $\eta_{c^{-}}$-related studies at Belle II and BESIII as discussed in sections III and IV. The displaced $e^{+} e^{-}$vertex searches can probe the $17 \mathrm{MeV} X$ boson in the region

$$
2.5 \times 10^{-4} \leq \varepsilon_{e} \leq 8.0 \times 10^{-4}
$$

with significance larger than 2 by assuming near-zero background, and it covers the $\varepsilon_{e}$ region preferred by Atomki. While we expect less than one signal event with the currently available Belle data sample of $1 \mathrm{ab}^{-1}$, we can start exploring the Atomki preferred region within a few years once Belle II accumulates data sample of $10 \mathrm{ab}^{-1}$ or more.

Our study with the displaced vertex is extended for wider mass range of $X$-like boson, whereby we determine the region of sensitivity with Belle II at $50 \mathrm{ab}^{-1}$, as displayed in figure 8. Two cases are considered: $B=0.1$ and $B=1$, where $B$ is the expected number of background events from the SM processes. We then use the Poisson probability of the expected background to fluctuate upward, to calculate the $p$-values and the corresponding 


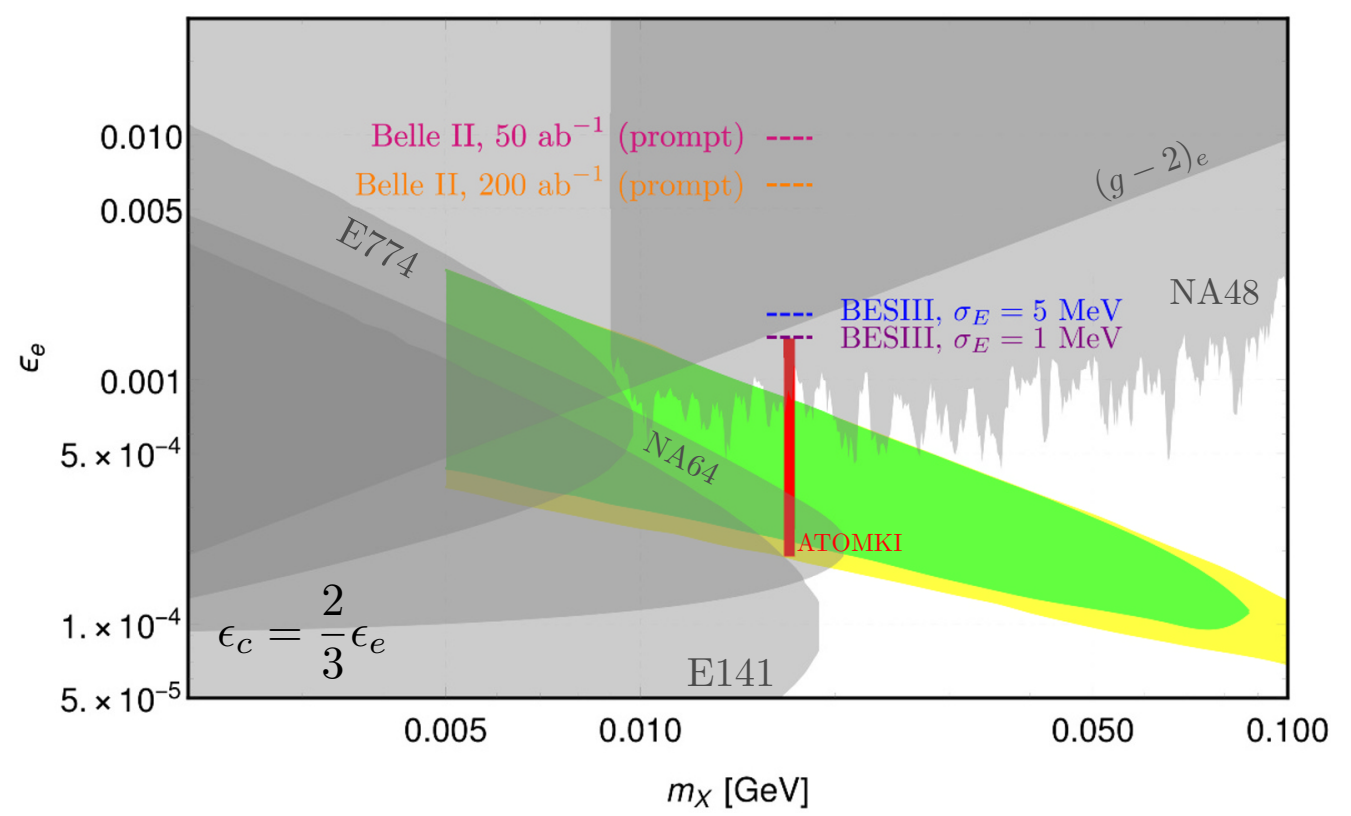

Figure 8. The yellow (green) contour corresponds $\geq 2 \sigma$ significance assuming SM background $B=0.1(B=1)$ from $e^{+} e^{-}$channel at Belle II with $50 \mathrm{ab}^{-1}$ luminosity, which probes rest of the favour parameter region of Atomki (red vertical band). The gray-shaded regions are excluded by current experiments relate to Xee coupling. Compare to the sensitivities of $e^{+} e^{-} \rightarrow \ell^{+} \ell^{-}+J / \Psi \rightarrow$ $\ell^{+} \ell^{-} e^{+} e^{-} \eta_{c}$ channel at Belle II and $e^{+} e^{-} \rightarrow J / \Psi \rightarrow e^{+} e^{-} \eta_{c}$ channel at BESIII by assuming the kinematic mixing between $X$ boson and photon, bringing the relation $\varepsilon_{c}=\frac{2}{3} \varepsilon_{e}$. For the future Belle II experiments 50, $200 \mathrm{ab}^{-1}$ in the $17 \mathrm{MeV}$ mass region, the significance bounds are denoted by magenta, orange dotted lines, respectively. In the case of BESIII for the energy resolutions $\sigma_{E}=$ $1 \mathrm{MeV}$ and $5 \mathrm{MeV}$, corresponding to section 3, they are denoted in purple and blue, respectively.

significances. The $2 \sigma$ significance region with $B=0.1(B=1)$ is shown as the yellow (green) contour in figure 8, which yields signal event $N_{S} \simeq 1\left(N_{S} \simeq 2\right){ }^{4}$ This study can probe the parameter region of $5 \mathrm{MeV} \leq m_{X} \leq 100 \mathrm{MeV}$ and $1.0 \times 10^{-4} \leq \varepsilon_{e} \leq 3 \times 10^{-3}$, which have not been constrained by any existing experiments. The upper edge of this region is determined by the condition $2 \mathrm{~mm} \leq d_{x y}$, while the lower edge is limited by the statistics. Therefore, with even higher luminosity of Belle II exceeding the target $50 \mathrm{ab}^{-1}$, the lower edge of the sensitivity region can be extended further.

\section{Summary and conclusion}

In summary, we propose several studies using $J / \Psi$ at lepton colliders such as Belle II and BESIII, to search for light vector boson around the mass range suggested by the ${ }^{8} \mathrm{Be}^{*}$ anomaly of the ATOMKI experiment. At BESIII, the $J / \Psi \rightarrow \eta_{c} X \rightarrow \eta_{c} e^{+} e^{-}$channel can be used to constrain the vector boson and charm quark coupling, $\varepsilon_{c}$. With the currently

\footnotetext{
${ }^{4}$ In this case, the $p$-value is obtained as $\sum_{n>N_{S}+B} P(n \mid B)$ which is the probability of upward fluctuation based on the SM-only hypothesis with $P(n \mid B)$ being the Poisson probability of observing $n$ events given $B$ events of expected background. Then the confidence level (CL) is CL $=1-p$. The $2 \sigma$ significance corresponds to $95.4 \%$ CL. For $B=1(0.1)$ events, we need $N_{S} \simeq 2(1)$ signal events to attain $2 \sigma$ significance.
} 
available sample of $N_{J / \Psi}=10^{10}$ and effective $\eta_{c}$ reconstruction efficiency of $1.23 \%$, we can exclude the region $\left|\varepsilon_{c}\right| \gtrsim 5 \times 10^{-3}$ for $m_{X}=17 \mathrm{MeV}$. If $N_{J / \Psi}=10^{11}$ is produced at BESIII in the near future, exclusion of the region $\left|\varepsilon_{c}\right| \simeq 3 \times 10^{-3}$ might be achieved. If universal coupling to up-type quarks is assumed, we expect that the entire favored signal region from the ${ }^{8} \mathrm{Be}^{*}$ anomaly could be covered.

On the other hand at Belle II, with higher CM energy of $10.59 \mathrm{GeV}$, we propose to study the process $e^{+} e^{-} \rightarrow \ell^{+} \ell^{-} J / \Psi$ followed by $J / \Psi \rightarrow \eta_{c} X \rightarrow \eta_{c} e^{+} e^{-}$. Using the recoil mass against $\ell^{+} \ell^{-} e^{+} e^{-}$, we perform MC study and find that the expected production of $J / \Psi$ events is about three orders of magnitude smaller than that of BESIII, thus yielding the sensitivity of $\left|\varepsilon_{c}\right| \gtrsim 1.8 \times 10^{-2}$ at $m_{X}=17 \mathrm{MeV}$.

Alternatively, we can study the process $e^{+} e^{-} \rightarrow X+J / \Psi \rightarrow e^{+} e^{-} \ell^{+} \ell^{-}$at Belle II and directly constrain the $X$ boson-electron coupling. The $X$ boson is boosted by the higher CM energy and heavy mass of $J / \Psi$, producing displaced vertex of $X \rightarrow e^{+} e^{-}$which is longer than several millimeters. Particularly, it is almost background free when the transverse flight distance is $2 \mathrm{~mm} \leq d_{x y} \leq 8 \mathrm{~mm}$. Selecting this window and requiring $>2 \sigma$ significance, it gives the sensitivity $2.0 \times 10^{-4} \leq\left|\varepsilon_{e}\right| \leq 8.0 \times 10^{-4}$ at $m_{X}=17 \mathrm{MeV}$ for $50 \mathrm{ab}^{-1}$ luminosity and covers most of the favored signal region from the claimed ${ }^{8} \mathrm{Be}^{*}$ anomaly. Extending the range of the $X$ boson mass, this method can cover the unprecedented parameter space of $9 \mathrm{MeV} \leq m_{X} \leq 100 \mathrm{MeV}$ and $1.0 \times 10^{-4} \leq\left|\varepsilon_{e}\right| \leq 10^{-3}$.

\section{Acknowledgments}

The work is supported in part by the National Research Foundation of Korea [NRF-2021R1A4A2001897 (SCP, YJK), NRF-2019R1A2C1089334 (SCP), and NRF2020R1I1A1A01066413 (PYT)].

Open Access. This article is distributed under the terms of the Creative Commons Attribution License (CC-BY 4.0), which permits any use, distribution and reproduction in any medium, provided the original author(s) and source are credited.

\section{References}

[1] Super-Kamiokande collaboration, Evidence for oscillation of atmospheric neutrinos, Phys. Rev. Lett. 81 (1998) 1562 [hep-ex/9807003] [INSPIRE].

[2] Muon G-2 collaboration, Final report of the muon E821 anomalous magnetic moment measurement at BNL, Phys. Rev. D 73 (2006) 072003 [hep-ex/0602035] [inSPIRE].

[3] A. Keshavarzi, D. Nomura and T. Teubner, $g-2$ of charged leptons, $\alpha\left(M_{Z}^{2}\right)$, and the hyperfine splitting of muonium, Phys. Rev. D 101 (2020) 014029 [arXiv:1911.00367] [INSPIRE].

[4] Planck collaboration, Planck 2018 results. VI. Cosmological parameters, Astron. Astrophys. 641 (2020) A6 [arXiv:1807.06209] [INSPIRE].

[5] D.Y. Cheong, S.M. Lee and S.C. Park, Primordial black holes in Higgs- $R^{2}$ inflation as the whole of dark matter, JCAP 01 (2021) 032 [arXiv:1912.12032] [INSPIRE]. 
[6] L. Canetti, M. Drewes and M. Shaposhnikov, Matter and antimatter in the universe, New J. Phys. 14 (2012) 095012 [arXiv: 1204.4186] [INSPIRE].

[7] Particle Data Group collaboration, Review of particle physics, PTEP 2020 (2020) 083C01 [INSPIRE].

[8] X.-G. He, G.C. Joshi, H. Lew and R.R. Volkas, Simplest Z' model, Phys. Rev. D 44 (1991) 2118 [INSPIRE].

[9] W. Altmannshofer, S. Gori, S. Profumo and F.S. Queiroz, Explaining dark matter and B decay anomalies with an $L_{\mu}-L_{\tau}$ model, JHEP 12 (2016) 106 [arXiv:1609.04026] [INSPIRE].

[10] P. Foldenauer, Light dark matter in a gauged $U(1)_{L_{\mu}-L_{\tau}}$ model, Phys. Rev. D 99 (2019) 035007 [arXiv: 1808.03647] [INSPIRE].

[11] S. Baek, N.G. Deshpande, X.G. He and P. Ko, Muon anomalous $g-2$ and gauged $L_{\mu}-L_{\tau}$ models, Phys. Rev. D 64 (2001) 055006 [hep-ph/0104141] [INSPIRE].

[12] Y. Kaneta and T. Shimomura, On the possibility of a search for the $L_{\mu}-L_{\tau}$ gauge boson at Belle-II and neutrino beam experiments, PTEP 2017 (2017) 053B04 [arXiv:1701.00156] [INSPIRE].

[13] T. Araki, S. Hoshino, T. Ota, J. Sato and T. Shimomura, Detecting the $L_{\mu}-L_{\tau}$ gauge boson at Belle II, Phys. Rev. D 95 (2017) 055006 [arXiv:1702.01497] [INSPIRE].

[14] Y. Jho, Y. Kwon, S.C. Park and P.-Y. Tseng, Search for muon-philic new light gauge boson at Belle II, JHEP 10 (2019) 168 [arXiv:1904.13053] [INSPIRE].

[15] A. Berlin, D. Hooper, G. Krnjaic and S.D. McDermott, Severely constraining dark matter interpretations of the $21 \mathrm{~cm}$ anomaly, Phys. Rev. Lett. 121 (2018) 011102 [arXiv: 1803.02804] [INSPIRE].

[16] W. Altmannshofer, S. Gori, M. Pospelov and I. Yavin, Quark flavor transitions in $L_{\mu}-L_{\tau}$ models, Phys. Rev. D 89 (2014) 095033 [arXiv:1403.1269] [INSPIRE].

[17] S. Shinohara, Search for the rare decay $K_{L} \rightarrow \pi^{0} \nu \bar{\nu}$ at J-PARC KOTO experiment, talk given at the International Conference on Kaon Physics 2019 (KAON2019), September 10-13, Perugia, Italy (2019).

[18] Y.C. Tung, 'Recent results from KOTO experiment, talk given at XXXIX International Symposium on Physics in Collision (PIC2019), September 16-20, Taipei, Taiwan (2019).

[19] C. Lin, Recent result on the measurement of $K_{L} \rightarrow \pi^{0} \nu \bar{\nu}$ at the J-PARC KOTO Experiment, talk given at the $3^{\text {rd }} J$-PARC Symposium, September 23-26, Tsukuba, Japan (2019).

[20] Y. Jho, S.M. Lee, S.C. Park, Y. Park and P.-Y. Tseng, Light gauge boson interpretation for $(g-2)_{\mu}$ and the $K_{L} \rightarrow \pi^{0}+$ (invisible) anomaly at the J-PARC KOTO experiment, JHEP 04 (2020) 086 [arXiv: 2001.06572] [INSPIRE].

[21] A.J. Krasznahorkay et al., Observation of anomalous internal pair creation in ${ }^{8}$ Be: a possible indication of a light, neutral boson, Phys. Rev. Lett. 116 (2016) 042501 [arXiv:1504.01527] [INSPIRE].

[22] A.J. Krasznahorkay et al., New evidence supporting the existence of the hypothetic X17 particle, arXiv:1910.10459 [INSPIRE].

[23] J.L. Feng et al., Protophobic fifth-force interpretation of the observed anomaly in ${ }^{8}$ Be nuclear transitions, Phys. Rev. Lett. 117 (2016) 071803 [arXiv:1604.07411] [INSPIRE]. 
[24] B. Fornal, Is there a sign of new physics in beryllium transitions?, Int. J. Mod. Phys. A 32 (2017) 1730020 [arXiv: 1707.09749] [INSPIRE].

[25] J.L. Feng, T.M.P. Tait and C.B. Verhaaren, Dynamical evidence for a fifth force explanation of the ATOMKI nuclear anomalies, Phys. Rev. D 102 (2020) 036016 [arXiv:2006.01151] [INSPIRE].

[26] NA48/2 collaboration, Search for the dark photon in $\pi^{0}$ decays, Phys. Lett. B 746 (2015) 178 [arXiv: 1504.00607] [inSPIRE].

[27] R. Essig et al., Working group report: new light weakly coupled particles, arXiv:1311.0029 [INSPIRE].

[28] H. Davoudiasl, H.-S. Lee and W.J. Marciano, Muon g - 2, rare kaon decays, and parity violation from dark bosons, Phys. Rev. D 89 (2014) 095006 [arXiv: 1402.3620] [INSPIRE].

[29] A.N. Khan, Global analysis of the source and detector nonstandard interactions using the short baseline $\nu$-e and $\bar{\nu}$-e scattering data, Phys. Rev. D 93 (2016) 093019 [arXiv: 1605.09284] [INSPIRE].

[30] L.-M. Gu, H.-B. Li, X.-X. Ma and M.-Z. Yang, Study of the electromagnetic Dalitz decays $\psi(\Upsilon) \rightarrow \eta_{c}\left(\eta_{b}\right) l^{+} l^{-}$, Phys. Rev. D 100 (2019) 016018 [arXiv:1904.06085] [InSPIRE].

[31] J. Fu, H.-B. Li, X. Qin and M.-Z. Yang, Study of the electromagnetic transitions $J / \psi \rightarrow P l^{+} l^{-}$and probe dark photon, Mod. Phys. Lett. A 27 (2012) 1250223 [arXiv:1111.4055] [INSPIRE].

[32] CLEO collaboration, $J / \psi$ and $\psi(2 S)$ radiative decays to $\eta(c)$, Phys. Rev. Lett. 102 (2009) 011801 [Erratum ibid. 106 (2011) 159903] [arXiv: 0805.0252] [INSPIRE].

[33] V.V. Anashin et al., Measurement of $J / \psi \rightarrow \eta_{c} \gamma$ at KEDR, Chin. Phys. C 34 (2010) 831 [arXiv: 1002.2071] [INSPIRE].

[34] D. Becirevic and F. Sanfilippo, Lattice QCD study of the radiative decays $J / \psi \rightarrow \eta_{c} \gamma$ and $h_{c} \rightarrow \eta_{c} \gamma$, JHEP 01 (2013) 028 [arXiv: 1206.1445] [INSPIRE].

[35] BeLLe II collaboration, The Belle II physics book, PTEP 2019 (2019) 123C01 [arXiv: 1808.10567] [INSPIRE].

[36] BESIII collaboration, Measurements of the branching fractions of $\eta_{c} \rightarrow K^{+} K^{-} \pi^{0}$, $K_{S}^{0} K^{ \pm} \pi^{\mp}, 2\left(\pi^{+} \pi^{-} \pi^{0}\right)$, and $p \bar{p}$, Phys. Rev. D 100 (2019) 012003 [arXiv:1903.05375] [INSPIRE].

[37] R. Barbieri and T.E.O. Ericson, Evidence against the existence of a low mass scalar boson from neutron-nucleus scattering, Phys. Lett. B 57 (1975) 270 [INSPIRE].

[38] J. Alwall et al., The automated computation of tree-level and next-to-leading order differential cross sections, and their matching to parton shower simulations, JHEP 07 (2014) 079 [arXiv: 1405.0301] [INSPIRE].

[39] A. Alloul, N.D. Christensen, C. Degrande, C. Duhr and B. Fuks, FeynRules 2.0 - A complete toolbox for tree-level phenomenology, Comput. Phys. Commun. 185 (2014) 2250 [arXiv: 1310.1921] [INSPIRE].

[40] BELLE II collaboration, Search for an invisibly decaying $Z^{\prime}$ boson at Belle II in $e^{+} e^{-} \rightarrow \mu^{+} \mu^{-}\left(e^{ \pm} \mu^{\mp}\right)$ plus missing energy final states, Phys. Rev. Lett. 124 (2020) 141801 [arXiv: 1912.11276] [INSPIRE]. 
[41] K. Hanagaki, H. Kakuno, H. Ikeda, T. Iijima and T. Tsukamoto, Electron identification in Belle, Nucl. Instrum. Meth. A 485 (2002) 490.

[42] V. Bertacchi et al., Track finding at Belle II, Comp. Phys. Commun. 259 (2021) 107610.

[43] M. Duerr, T. Ferber, C. Hearty, F. Kahlhoefer, K. Schmidt-Hoberg and P. Tunney, Invisible and displaced dark matter signatures at Belle II, JHEP 02 (2020) 039 [arXiv:1911.03176] [INSPIRE]. 